J. Pestic. Sci., 31(3), 311-315 (2006)

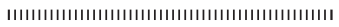

Original Article

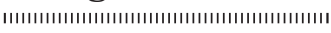

\title{
Synthesis of (+)-(S)-isorobinal together with its antipod, a cyclic monoterpene functioning as the sex pheromone of Rhizoglyphus setosus and its distribution among Astigmata*
}

\author{
Nobuhiro Shimizu, Atsuko Mizoguchi, Kenjiro Murakami, Koji Noge, Naoki Mori, \\ Ritsuo NisHIDA and Yasumasa KUWAHARA** \\ Division of Applied Life Sciences, Graduate School of Agriculture, Kyoto University, Kyoto 606-8502, Japan
}

(Received September 7, 2005; Accepted February 24, 2006)

\begin{abstract}
Both compounds, $(+)-(S)$ - and (-)-(R)-4-isopropenyl-3-oxo-1-cyclohexene-1-carbaldehyde $[(+)-(S)$ - and $(-)-$ $(R)$-isorobinal, $(S)$ - and $(R)-1,[\alpha]_{\mathrm{D}}^{28}+51^{\circ}\left(c=1.0, \mathrm{CHCl}_{3}\right)$ and $[\alpha]_{\mathrm{D}}^{25}-45^{\circ}\left(c=1.3, \mathrm{CHCl}_{3}\right), 95 \% e e$ and $\left.88 \% e e\right]$, were synthesized in seven steps starting from $(R)$ - and $(S)$-perillyl alcohol [(R)- and $(S)$-2] available commercially. Isorobinal (1) is distributed among seven species of Astigmata among 61 species examined. $(S)$-Isorobinal $[(S)-1]$, known as the female sex pheromone of Rhizoglyphus setosus, was confirmed in two other Rhizoglyphus species, while its biological function remained obscure against each species. In the other four species, not only its configuration but also its function has not yet been determined. (C) Pesticide Science Society of Japan
\end{abstract}

Keywords: mite, Astigmata, Rhizoglyphus sp., isorobinal, 4-isopropenyl-3-oxo-1-cyclohexene-1-carbaldehyde.

\section{Introduction}

A total of 88 compounds, classified into 27 compounds with monoterpenoid carbon skeletons, two sesquiterpenes, eight aromatics, and 24 aliphatic compounds (a ketone, aldehydes, fatty acid esters and alkyl formates) together with 27 aliphatic hydrocarbons have been demonstrated as components of the opisthonotal gland secretions from 61 species of mites belonging to ten families of Astigmata in Acari. ${ }^{1)}$ Parts of the compounds are known to function either as an alarm pheromone, an aggregation pheromone or a sex pheromone in each corresponding species. Among 27 monoterpenoids, ten compounds were first isolated and identified from Astigmata, with the structures unequivocally confirmed by synthesis. ${ }^{1)}$

Isorobinal [4-isopropenyl-3-oxo-1-cyclohexene-1-carbaldehyde, 1], one of such novel cyclic monoterpenes, has been identified from the secretion of the astigmatid mite Rhizoglyphus sp. "oki", [its ITS-II (internal transcriptive spacer-II) re-

* Chemical Ecology of Astigmatid Mites, Part LXXIX.

** To whom correspondence should be addressed: E-mail: kuwahara@ kyotogakuen.ac.jp, Fax: +81-771-29-3641 Present address: Kyotogakuen University, Kameoka-shi, Kyoto $621-8555$

(C) Pesticide Science Society of Japan

Abbreviations: MCPBA, meta-chloroperbenzoic acid; PCC, pyridinium chlorochromate. gion consists of 460 bps (code name RH 6, GenBank Accession Number: AB104931)]. ${ }^{3)}$ (S)-Isomer of $\mathbf{1}$ has been demonstrated as the female sex pheromone of the related mite, Rhizoglyphus setosus (Acari: Acaridae), ${ }^{4)}$ using both enantiomers of $\mathbf{1}$ prepared by a group from Tohoku University $^{5)}$ and also by us. Herein we report the syntheses of both enantiomers to determine the stereochemistry and the natural distribution of $\mathbf{1}$ among Astigmata.

\section{Methods and Materials}

\section{Analytical methods}

GC/MS was carried out using a Hewlett Packard HP-5989B gas chromatograph/mass spectrometer operated at $70 \mathrm{eV}$ in the split-less mode, with an HP-5 MS capillary column $(0.25$ $\mathrm{mm}$ in diameter $\times 30 \mathrm{~m}$ in length, $0.25 \mu \mathrm{m}$ in film thickness). Helium was used as the carrier gas at $1.23 \mathrm{ml} / \mathrm{min}$, at temperatures programmed from $60^{\circ} \mathrm{C}$ to $290^{\circ} \mathrm{C}$ at $10^{\circ} \mathrm{C} / \mathrm{min}$, with an initial 2-min hold at $60^{\circ} \mathrm{C}$ and then at $290^{\circ} \mathrm{C}$ for $5 \mathrm{~min} .{ }^{1} \mathrm{H}$ and ${ }^{13} \mathrm{C}$-NMR spectra were obtained with a Bruker AC300 instrument $\left({ }^{1} \mathrm{H}\right.$ at $300 \mathrm{MHz},{ }^{13} \mathrm{C}$ at $\left.75 \mathrm{MHz}\right)$ in $\mathrm{CDCl}_{3}$ solution with TMS as an internal standard. UV spectra were measured with a Beckman DU-64 spectrophotometer. Optical rotation values were measured with a Jasco DIP-370 spectropolarimeter. 
2. HPLC analyses of (+)-(S)-isorobinal and (-)-(R)isorobinal [(S)-1 and $(R)-1]$

Compounds $(S)$ - and $(R)-\mathbf{1}$ were chromatographed in a chiral HPLC column [Chiralpak AD-H (Daicel Chemical Ind., Ltd.), $250 \mathrm{~mm} \times 4.6 \mathrm{~mm}$ i.d.], and the column was eluted with hexane-isopropyl alcohol $(200: 1)$ at a flow rate of $1 \mathrm{ml} / \mathrm{min}$. Peaks were detected by UV at $254 \mathrm{~nm}$ using a Jasco UVIDEC-100-H, and recorded with a HP 3390 Integrator. In order to determine the stereochemistry of $\mathbf{1}$ from three Rhizoglyphus species, hexane extracts were prepared by dipping mite bodies in hexane for three minutes. Each mite species, weight of mites and extracted volume of solvent was as follows: Rhizoglyphus sp. "oki”, $778 \mathrm{mg}$ in hexane $2.6 \mathrm{ml}$, Rhizoglyphus sp. "mori", $579 \mathrm{mg}$ in hexane $2.0 \mathrm{ml}$, and R. setosus, $97 \mathrm{mg}$ in hexane $0.5 \mathrm{ml}$.

3. Syntheses of (+)-(S)-isorobinal and (-)-(R)-isorobinal $[(S)-1$ and $(R)-1]$

3.1. (R)-1,2-Epoxy-8-p-menthen-7-ol [(R)-3]

To a stirred and ice-cooled solution of MCPBA $(70 \%, 1.77 \mathrm{~g}$, $7.20 \mathrm{mmol})$ in $\mathrm{CH}_{2} \mathrm{Cl}_{2}(30 \mathrm{ml})$ was added $(R)-2(1.00 \mathrm{~g}$, $6.58 \mathrm{mmol})$ in $\mathrm{CH}_{2} \mathrm{Cl}_{2}(5 \mathrm{ml})$. The mixture was stirred overnight at room temperature. It was then quenched with sat. aq. $\mathrm{Na}_{2} \mathrm{~S}_{2} \mathrm{O}_{3}$ and the product was extracted with EtOAc. The organic layer was washed with sat. aq. $\mathrm{NaHCO}_{3}$, water and brine, dried over $\mathrm{Na}_{2} \mathrm{SO}_{4}$, and concentrated in vacuo. The residue was purified by silica gel column chromatography (eluting with hexane-EtOAc, $2: 1$ ) to give $1.10 \mathrm{~g}$ (quantitative) of $(R)-\mathbf{3}$ as a diastereomeric mixture. GC/MS $t_{\mathrm{R}} 11.12$ $\min$ and $11.25 \mathrm{~min} ;[\alpha]_{\mathrm{D}}^{25}+49^{\circ}\left(c=1.0, \mathrm{CHCl}_{3}\right) ; \mathrm{MS} m / z(\%)$ : $168\left(\mathrm{M}^{+}, 2\right), 150$ (47), 137 (40), 121 (71), 107 (66), 93 (78), 79 (100), 67 (79), 55 (45), 41 (64). MS m/z (\%): $168\left(\mathrm{M}^{+}, 2\right)$, 150 (24), 135 (22), 121 (29), 110 (53), 93 (83), 79 (100), 67 (79), 55 (55), $41(64) ;{ }^{1} \mathrm{H}-\mathrm{NMR} \delta\left(\mathrm{CDCl}_{3}\right)$ as a diastereomeric mixture: 1.6-2.0 (6H, m), $1.71\left(3 \mathrm{H}, \mathrm{s},=\mathrm{CC}_{3}\right)$, 2.0-2.2 (1H, m, $\left.\mathrm{CH}_{2} \mathrm{CHCH}_{2}\right), 3.35\left(1 \mathrm{H}, \mathrm{m}, \mathrm{CH}_{2} \mathrm{CHOC}\right)$, 3.56-3.76 $\left(2 \mathrm{H}, \mathrm{m}, \mathrm{CH}_{2} \mathrm{OH}\right), 4.68\left(1 \mathrm{H}, \mathrm{s},=\mathrm{CH}_{2}\right), 4.74(1 \mathrm{H}, \mathrm{t}-$ like, $\left.J=1.4 \mathrm{~Hz},=\mathrm{CH}_{2}\right) ;{ }^{13} \mathrm{C}-\mathrm{NMR} \delta\left(\mathrm{CDCl}_{3}\right)$ as a diastereomeric mixture: $20.9\left(\mathrm{CH}_{3}\right), 24.7\left(\mathrm{CH}_{2}\right), 25.9\left(\mathrm{CH}_{2}\right), 30.2$ $\left(\mathrm{CH}_{2}\right), 36.8(\mathrm{CH}), 56.7(\mathrm{CH}), 59.9(\mathrm{C}), 64.1\left(\mathrm{CH}_{2}\right), 109.2$ $\left(\mathrm{CH}_{2}\right), 148.7(\mathrm{C})$.

3.2. (S)-1,2-Epoxy-8-p-menthen-7-ol [(S)-3]

In the same manner as described above, $(S)-2$ (1.00 g, $6.58 \mathrm{mmol})$ gave $(S)-3(983 \mathrm{mg}, 89 \%)$ as a mixture of two diastereomers. Its $\mathrm{GC} / \mathrm{MS},{ }^{1} \mathrm{H}-$ and ${ }^{13} \mathrm{C}-\mathrm{NMR}$ spectra were identical to those of the $R$ isomer. $[\alpha]_{\mathrm{D}}^{25}-45^{\circ}(c=1.0$, $\mathrm{CHCl}_{3}$ )

\section{3. (R)-2-(Phenylseleno)-8-p-menthen-1,7-diol [(R)-4]}

To a stirred solution of diphenyl diselenide (2.16 g, $6.92 \mathrm{mmol})$ in dry EtOH $(42 \mathrm{ml})$ was added $\mathrm{NaBH}_{4}(0.524 \mathrm{~g}$, $13.9 \mathrm{mmol}$ ) under an $\mathrm{N}_{2}$ atmosphere. The mixture turned colorless, and $(R)-3(1.10 \mathrm{~g}, 6.55 \mathrm{mmol})$ in dry EtOH $(6 \mathrm{ml})$ was then added dropwise. The mixture was refluxed for $2 \mathrm{hr}$ with stirring under $\mathrm{N}_{2}$ atmosphere. After quenching with $0.5 \mathrm{~N}$
$\mathrm{HCl}$, the mixture was extracted with EtOAc. The organic layer was washed with sat. aq. $\mathrm{NaHCO}_{3}$, water, brine and dried over $\mathrm{Na}_{2} \mathrm{SO}_{4}$, and concentrated in vacuo. The residue was purified by silica gel column chromatography (eluting with hexaneEtOAc, $4: 1)$ to give $(R)-4(1.23 \mathrm{~g}, 58 \%)$ and the isomer $(R)$ 1-(phenylseleno)-8-p-menthen-2,7-diol [ $(R)-5 ; 0.32 \mathrm{~g}, 35 \%]$.

$(R)-4: \mathrm{GC} / \mathrm{MS} t_{\mathrm{R}} 21.10 \mathrm{~min} ;[\alpha]_{\mathrm{D}}^{29}+59^{\circ}\left(c=1.0, \mathrm{CHCl}_{3}\right)$; MS $m / z(\%): 326\left(\mathrm{M}^{+}, 18\right), 308$ (5), 169 (32), 151 (100), 133 (42), 123 (41), 107 (53), 93 (48), 81 (50), 67 (52), 55 (45), 41 (41); ${ }^{1} \mathrm{H}-\mathrm{NMR} \delta\left(\mathrm{CDCl}_{3}\right)$ as a diastereomeric mixture: 1.6-2.4 (7H, m), $1.71\left(3 \mathrm{H}, \mathrm{s},=\mathrm{CCH}_{3}\right), 3.3-3.5(1 \mathrm{H}, \mathrm{m}$, $\mathrm{CH}_{2} \mathrm{CHSePh}$ ), $3.52\left(1 \mathrm{H}, \mathrm{d}, J=11.4 \mathrm{~Hz}, \mathrm{CH}_{2} \mathrm{OH}\right), 3.88(1 \mathrm{H}, \mathrm{d}$, $\left.J=11.4 \mathrm{~Hz}, \mathrm{CH}_{2} \mathrm{OH}\right), 4.73\left(2 \mathrm{H}, \mathrm{s},=\mathrm{CH}_{2}\right), 7.2-7.7(5 \mathrm{H}, \mathrm{m}$, $\mathrm{Ph}) ;{ }^{13} \mathrm{C}-\mathrm{NMR} \delta\left(\mathrm{CDCl}_{3}\right)$ as a diastereomeric mixture: 21.1 $\left(\mathrm{CH}_{3}\right), 25.7\left(\mathrm{CH}_{2}\right), 30.0\left(\mathrm{CH}_{2}\right), 33.2\left(\mathrm{CH}_{2}\right), 40.5(\mathrm{CH}), 50.0$ $(\mathrm{CH}), 69.7\left(\mathrm{CH}_{2}\right), 73.8(\mathrm{C}), 109.0\left(\mathrm{CH}_{2}\right), 127.8(\mathrm{Ph}), 129.3$ (Ph), $133.9(\mathrm{Ph}), 134.3(\mathrm{Ph}), 149.3(\mathrm{C})$.

$(R)-5: \mathrm{GC} / \mathrm{MS} t_{\mathrm{R}} 19.69 \mathrm{~min} ;[\alpha]_{\mathrm{D}}^{25}+39^{\circ}\left(c=1.0, \mathrm{CHCl}_{3}\right)$; MS $m / z(\%): 308\left(\mathrm{M}^{+}-18,100\right), 158$ (60), 150 (81), 133 (52), 109 (62), 93 (74), 81 (53), 67 (60), 55 (53), 41 (57); ${ }^{1} \mathrm{H}-\mathrm{NMR}$ $\delta\left(\mathrm{CDCl}_{3}\right)$ as a diastereomeric mixture: $1.6-2.4(7 \mathrm{H}, \mathrm{m}), 1.77$ $\left(3 \mathrm{H}, \mathrm{s},=\mathrm{CCH}_{3}\right), 3.74\left(1 \mathrm{H}, \mathrm{d}, J=11.1 \mathrm{~Hz}, \mathrm{CH}_{2} \mathrm{OH}\right), 4.09(1 \mathrm{H}$, d, $\left.J=11.1 \mathrm{~Hz}, \underline{\mathrm{C}}_{2} \mathrm{OH}\right), 4.13\left(1 \mathrm{H}, \mathrm{m}, \mathrm{CH}_{2} \mathrm{C} \underline{\mathrm{HOH}}\right), 4.77(2 \mathrm{H}$, $\left.\mathrm{s},=\mathrm{C}_{2}\right), 7.3-7.6(5 \mathrm{H}, \mathrm{m}, \mathrm{Ph})$.

3.4. (S)-2-(Phenylseleno)-8-p-menthen-1,7-diol [(S)-4] In the same manner as described above, $(S)-3 \quad(2.56 \mathrm{~g}$, $15.3 \mathrm{mmol})$ yielded $(S)-4(2.84 \mathrm{~g}, 57 \%)$. Its GC/MS, ${ }^{1} \mathrm{H}-$ and ${ }^{13} \mathrm{C}$-NMR spectra were identical with those of the $R$ isomer. $[\alpha]_{\mathrm{D}}^{29}-56^{\circ}\left(c=1.0, \mathrm{CHCl}_{3}\right)$.

3.5. (R)-7-Acetoxy-2-(phenylseleno)-8-p-menthen-1-ol [(R)-6]

Acetic anhydride $(3.5 \mathrm{ml})$ was added to a stirred and icecooled solution of $(R)-4(1.23 \mathrm{~g}, 3.77 \mathrm{mmol})$ in dry pyridine $(5 \mathrm{ml})$. The mixture was stirred for $4 \mathrm{hr}$ at room temperature, then poured into ice water, and extracted with $\mathrm{Et}_{2} \mathrm{O}$. The ethereal extract was washed successively with $0.5 \mathrm{NHCl}$, water, aq. sat. $\mathrm{NaHCO}_{3}$ and brine, dried over $\mathrm{Na}_{2} \mathrm{SO}_{4}$ and concentrated in vacuo. The residue was purified by silica gel column chromatography (eluting with hexane-EtOAc, $5: 1$ ) to give $(R)-6(1.11 \mathrm{~g}, 80 \%)$ as an oil. GC/MS $t_{\mathrm{R}} 21.82 \mathrm{~min} ;[\alpha]_{\mathrm{D}}^{27}$ $+33^{\circ}\left(c=1.0, \mathrm{CHCl}_{3}\right)$; $\mathrm{MS} m / z(\%): 368\left(\mathrm{M}^{+}, 9\right), 211$ (7), 193 (41), 151 (33), 133 (100), 107 (24), 91 (22), 81 (16), 67 (14), 55 (14), 43 (61); ${ }^{1} \mathrm{H}-\mathrm{NMR} \delta\left(\mathrm{CDCl}_{3}\right): 1.6-2.4(7 \mathrm{H}, \mathrm{m})$, $1.73\left(3 \mathrm{H}, \mathrm{s},=\mathrm{CCH}_{3}\right), 1.78\left(3 \mathrm{H}, \mathrm{s}, \mathrm{O}=\mathrm{CCH}_{3}\right), 3.50(1 \mathrm{H}, \mathrm{dd}$, $\left.J=5.1, \quad 3.0 \mathrm{~Hz}, \mathrm{CH}_{2} \mathrm{C} \underline{\mathrm{HSePh}}\right), 4.05(1 \mathrm{H}, \mathrm{d}, J=11.3 \mathrm{~Hz}$, $\left.\mathrm{CH}_{2} \mathrm{OAc}\right), 4.41\left(1 \mathrm{H}, \mathrm{d}, J=11.3 \mathrm{~Hz}, \mathrm{C}_{2} \mathrm{OAc}\right), 4.74(2 \mathrm{H}$, $\left.\mathrm{s},=\mathrm{C}_{2}\right), 7.3-7.7(5 \mathrm{H}, \mathrm{m}, \mathrm{Ph}) ;{ }^{13} \mathrm{C}-\mathrm{NMR} \delta\left(\mathrm{CDCl}_{3}\right): 20.4$ $\left(\mathrm{CH}_{3}\right), 21.1\left(\mathrm{CH}_{3}\right), 25.3\left(\mathrm{CH}_{2}\right), 30.2\left(\mathrm{CH}_{2}\right), 33.8\left(\mathrm{CH}_{2}\right), 40.6$ $(\mathrm{CH}), 50.6(\mathrm{CH}), 71.5\left(\mathrm{CH}_{2}\right), 73.0(\mathrm{CH}), 109.1\left(\mathrm{CH}_{2}\right), 127.6$ (Ph), $129.1(\mathrm{Ph}), 134.3(\mathrm{Ph}), 134.5(\mathrm{Ph}), 149.8(\mathrm{C}), 170.8$ $\left(\mathrm{CH}_{3} \mathrm{C}=\mathrm{O}\right)$.

3.6. (S)-7-Acetoxy-2-(phenylseleno)-8-p-menthen-1-ol [(S)-6]

In the same manner as described above, $(S)-4$ (1.00 g, 
$3.07 \mathrm{mmol}$ ) gave $(S)-6(970 \mathrm{mg}, 86 \%)$. Its GC/MS, ${ }^{1} \mathrm{H}-$ and

${ }^{13} \mathrm{C}$-NMR spectra were identical to those of the $S$ isomer. $[\alpha]_{\mathrm{D}}^{28}-22^{\circ}\left(c=1.0, \mathrm{CHCl}_{3}\right)$.

\section{7. (R)-7-Acetoxy-2,8-p-menthadien-1-ol [(R)-7]}

To the stirred and ice-cooled solution of $(R)-6(732 \mathrm{mg}$, $1.99 \mathrm{mmol})$ in THF $(45 \mathrm{ml})$ was added $30 \% \mathrm{H}_{2} \mathrm{O}_{2}(3.69 \mathrm{ml}$, $32.4 \mathrm{mmol}$ ) dropwise. The resulting solution was stirred for $20 \mathrm{~min}$ and then allowed to come to room temperature with stirring for $2 \mathrm{hr}$. After the addition of water, the mixture was extracted with EtOAc. The organic layer was washed with water and brine, dried over $\mathrm{Na}_{2} \mathrm{SO}_{4}$, and concentrated in vacuo. The residue was purified by silica gel column chromatography (eluting with hexane-EtOAc, $4: 1$ ) to give $(R)-7$ $(300 \mathrm{mg}, 72 \%)$ as an oil. GC/MS $t_{\mathrm{R}} 13.03 \mathrm{~min} ;[\alpha]_{\mathrm{D}}^{27}+84^{\circ}$ $\left(c=1.0, \mathrm{CHCl}_{3}\right)$; $\mathrm{MS} \mathrm{m} / z(\%): 192\left(\mathrm{M}^{+}-18,2\right), 150$ (14), 137 (100), 109 (78), 95 (33), 79 (21), 67 (10), 55 (12), 43 (50); ${ }^{1} \mathrm{H}-\mathrm{NMR} \delta\left(\mathrm{CDCl}_{3}\right): 1.6-1.9(4 \mathrm{H}, \mathrm{m}), 1.75\left(3 \mathrm{H}, \mathrm{s},=\mathrm{CCH}_{3}\right)$, $2.12\left(3 \mathrm{H}, \mathrm{s}, \mathrm{O}=\mathrm{CC}_{3}\right), 2.70\left(1 \mathrm{H}, \mathrm{m}, \mathrm{CH}_{2} \mathrm{CHCH}=\right), 4.00$ $\left(1 \mathrm{H}, \mathrm{d}, J=11.2 \mathrm{~Hz}, \mathrm{CH}_{2} \mathrm{OAc}\right), 4.09(1 \mathrm{H}, \mathrm{d}, J=11.2 \mathrm{~Hz}$, $\left.\mathrm{CH}_{2} \mathrm{OAc}\right), 4.76\left(1 \mathrm{H}\right.$, br. $\left.\mathrm{s},=\mathrm{CH}_{2}\right), 4.80(1 \mathrm{H}, \mathrm{t}$-like, $J=1.5$ $\left.\mathrm{Hz},=\mathrm{C}_{2}\right), 5.73(1 \mathrm{H}, \mathrm{ddd}, J=10.2,2.4,1.1 \mathrm{~Hz}, \mathrm{CH}=\mathrm{C} \underline{\mathrm{HCH}})$, $5.85(1 \mathrm{H}$, ddd, $J=10.2,2.4,0.6 \mathrm{~Hz}, \mathrm{CH}=\mathrm{CHCH})$.

\section{8. (S)-7-Acetoxy-2,8-p-menthadien-1-ol [(S)-7]}

In the same manner as described above, $(S)-6$ (970 mg, $2.64 \mathrm{mmol}$ ) yielded $(S)-7$ (426 mg, 77\%). Its GC/MS, ${ }^{1} \mathrm{H}$ - and ${ }^{13} \mathrm{C}$-NMR spectra were identical to those of the $S$ isomer. $[\alpha]_{\mathrm{D}}^{26}-70^{\circ}\left(c=1.0, \mathrm{CHCl}_{3}\right)$.

\section{9. (S)-7-Acetoxy-3-oxo-1,8-p-menthadiene $[(S)-8]$}

To the stirred solution of PCC $(1.09 \mathrm{~g}, 4.95 \mathrm{mmol})$ in dry $\mathrm{CH}_{2} \mathrm{Cl}_{2}(22 \mathrm{ml})$ was added $(R)-7(300 \mathrm{mg}, 1.43 \mathrm{mmol})$ in dry $\mathrm{CH}_{2} \mathrm{Cl}_{2}(5 \mathrm{ml})$. The mixture was stirred at room temperature overnight, then diluted with $\mathrm{Et}_{2} \mathrm{O}$ and filtered through Celite. The filtrate was concentrated in vacuo and the residue was purified by silica gel column chromatography (eluting with hexane-EtOAc, $5: 1)$ to give $(S)-8(232 \mathrm{mg}, 78 \%)$ as an oil. GC/MS $t_{\mathrm{R}} 14.54 \mathrm{~min} ;[\alpha]_{\mathrm{D}}^{27}+29^{\circ}\left(c=1.0, \mathrm{CHCl}_{3}\right) ; \mathrm{MS} m / z$ (\%): $208\left(\mathrm{M}^{+}, 7\right), 193$ (3), 166 (5), 148 (22), 133 (10), 120 (9), 105 (5), 98 (100), $43(28) ;{ }^{1} \mathrm{H}-\mathrm{NMR} \delta\left(\mathrm{CDCl}_{3}\right): 1.76(3 \mathrm{H}$, $\left.\mathrm{s},=\mathrm{CCH}_{3}\right), 2.0-2.2\left(2 \mathrm{H}, \mathrm{m}, \mathrm{CH}_{2} \mathrm{CH}_{2} \mathrm{CH}\right), 2.14$ (3H, s, $\left.\mathrm{O}=\mathrm{CCH}_{3}\right), 2.35\left(2 \mathrm{H}, \mathrm{td}, J=5.0,0.7 \mathrm{~Hz}, \mathrm{CH}_{2} \underline{\mathrm{C}}_{2} \mathrm{C}=\right), 3.03$ $\left(1 \mathrm{H}, \mathrm{dd}, J=10.3,5.3 \mathrm{~Hz}, \quad \mathrm{CH}_{2} \mathrm{CHC}=\mathrm{O}\right), 4.68(2 \mathrm{H}, \mathrm{d}$, $\left.J=0.7 \mathrm{~Hz}, \mathrm{C}_{2} \mathrm{OAc}\right), 4.77\left(1 \mathrm{H}, \mathrm{d}, J=0.5 \mathrm{~Hz},=\underline{\mathrm{CH}}_{2}\right), 4.97$ $\left(1 \mathrm{H}, \quad \mathrm{t}\right.$-like, $\left.J=1.5 \mathrm{~Hz},=\mathrm{C}_{2}\right), 6.05(1 \mathrm{H}, \mathrm{t}, J=1.5 \mathrm{~Hz}$, $\mathrm{C}=\mathrm{C} \underline{\mathrm{HC}}=\mathrm{O}) ;{ }^{13} \mathrm{C}-\mathrm{NMR} \delta\left(\mathrm{CDCl}_{3}\right): 20.6\left(\mathrm{CH}_{3}\right), 20.7\left(\mathrm{CH}_{3}\right)$, $25.7\left(\mathrm{CH}_{2}\right), 27.4\left(\mathrm{CH}_{2}\right), 54.4(\mathrm{CH}), 64.8\left(\mathrm{CH}_{2}\right), 113.9\left(\mathrm{CH}_{2}\right)$, $124.6(\mathrm{CH}), 142.9(\mathrm{C}), 157.6(\mathrm{C}), 170.2\left(\mathrm{CH}_{3} \mathrm{C}=\mathrm{O}\right), 198.9$ $(\mathrm{C}=\mathrm{O})$.

3.10. (R)-7-Acetoxy-3-oxo-1,8-p-menthadiene [(R)-8]

In the same manner as described above, $(S)-7$ (208 mg, $0.99 \mathrm{mmol})$ gave $(R)-8(158 \mathrm{mg}, 77 \%)$. Its GC/MS, ${ }^{1} \mathrm{H}-$ and ${ }^{13} \mathrm{C}$-NMR spectra were identical to those of the $S$ isomer. $[\alpha]_{\mathrm{D}}^{26}-28^{\circ}\left(c=1.0, \mathrm{CHCl}_{3}\right)$.

3.11. (S)-3-Oxo-1,8-p-menthadien-7-ol [(S)-9]

A catalytic amount of $\mathrm{K}_{2} \mathrm{CO}_{3}$ was added to a solution of $(S)-8$
(97 mg, $0.47 \mathrm{mmol})$ in $\mathrm{MeOH}(20 \mathrm{ml})$ at $-15^{\circ} \mathrm{C}$, and stirred for $20 \mathrm{~min}$. After the addition of water, the mixture was neutralized with $0.5 \mathrm{M} \mathrm{HCl}$, then diluted with water, and extracted with EtOAc. The organic layer was washed with brine, dried over $\mathrm{Na}_{2} \mathrm{SO}_{4}$ and concentrated in vacuo. The residual oily product was purified by silica gel column chromatography (eluting with hexane- $\left.\mathrm{Et}_{2} \mathrm{O}, 1: 1\right)$ to give $(S)-9(66 \mathrm{mg}$, $85 \%)$. GC/MS $t_{\mathrm{R}} 13.68 \mathrm{~min} ;[\alpha]_{\mathrm{D}}^{26}+24^{\circ}\left(c=1.0, \mathrm{CHCl}_{3}\right)$; $\mathrm{MS}$ m/z (\%): $166\left(\mathrm{M}^{+}, 33\right), 151$ (40), 135 (32), 121 (7), 107 (9), 98 (100), 91 (14), 67 (36), 55 (19), 42 (31); ${ }^{1} \mathrm{H}-\mathrm{NMR} \delta$ $\left(\mathrm{CDCl}_{3}\right): 1.75\left(3 \mathrm{H}, \mathrm{s},=\mathrm{CCH}_{3}\right), 2.0-2.2\left(2 \mathrm{H}, \mathrm{m}, \mathrm{CH}_{2} \mathrm{CH}_{2} \mathrm{CH}\right)$, $2.32\left(2 \mathrm{H}, \mathrm{t}, J=5.7 \mathrm{~Hz}, \mathrm{CH}_{2} \mathrm{CH}_{2} \mathrm{C}=\right), 3.02(1 \mathrm{H}, \mathrm{dd}, J=10.0$, $\left.5.4 \mathrm{~Hz}, \mathrm{CH}_{2} \mathrm{CHC}=\mathrm{O}\right), 4.26\left(2 \mathrm{H}, \mathrm{s}, \mathrm{CH}_{2} \mathrm{OH}\right), 4.76(1 \mathrm{H}$, $\left.\mathrm{s},=\mathrm{C}_{2}\right), 4.96\left(1 \mathrm{H}, \mathrm{t}\right.$-like, $\left.J=1.5 \mathrm{~Hz},=\mathrm{CH}_{2}\right), 6.16(1 \mathrm{H}, \mathrm{t}$, $J=1.6 \mathrm{~Hz}, \mathrm{C}=\mathrm{CHC}=\mathrm{O}) ;{ }^{13} \mathrm{C}-\mathrm{NMR} \delta\left(\mathrm{CDCl}_{3}\right): 20.6\left(\mathrm{CH}_{3}\right)$, $25.4\left(\mathrm{CH}_{2}\right), 27.6\left(\mathrm{CH}_{2}\right), 54.6(\mathrm{CH}), 64.7\left(\mathrm{CH}_{2}\right), 113.8\left(\mathrm{CH}_{2}\right)$, $123.2(\mathrm{CH}), 143.1(\mathrm{C}), 163.6(\mathrm{C}), 199.6(\mathrm{C}=\mathrm{O})$.

3.12. (R)-3-Oxo-1,8-p-menthadien-7-ol [(R)-9]

In the same manner as described above, $(R)-8$ (131 mg, $0.63 \mathrm{mmol}$ ) yielded $(R)-9$ (95 mg, 91\%). Its GC/MS, ${ }^{1} \mathrm{H}$ - and ${ }^{13} \mathrm{C}$-NMR spectra were identical to those of the $S$ isomer. $[\alpha]_{\mathrm{D}}^{25}-25^{\circ}\left(c=1.0, \mathrm{CHCl}_{3}\right)$.

3.13. (1)-(S)-Isorobinal [(S)-1]

To a solution of $(S)-9(66 \mathrm{mg}, 0.40 \mathrm{mmol})$ in anhydrous $\mathrm{CH}_{2} \mathrm{Cl}_{2}$ (20 ml), Dess-Martin periodinane (296 mg, $0.70 \mathrm{mmol}$ ) and 3 drops of pyridine were added at $0^{\circ} \mathrm{C}$. The mixture was stirred for $15 \mathrm{~min}$, and then diluted with $\mathrm{Et}_{2} \mathrm{O}$. After the addition of sat. aq. $\mathrm{NaHCO}_{3}$ and $\mathrm{Na}_{2} \mathrm{~S}_{2} \mathrm{O}_{3}$ to the mixture under vigorously stirring, the resulting solution was extracted with $\mathrm{Et}_{2} \mathrm{O}$, and the ethereal solution of the product was successively washed with water and brine, dried over $\mathrm{Na}_{2} \mathrm{SO}_{4}$ and concentrated in vacuo. The residue was chromatographed on a silica gel column (eluting with hexane$\left.\mathrm{Et}_{2} \mathrm{O}, 3: 1\right)$ to give $(S)-\mathbf{1}(44 \mathrm{mg}, 68 \%)$. GC/MS $t_{\mathrm{R}}=10.97 \mathrm{~min}$; $[\alpha]_{\mathrm{D}}^{28}+51^{\circ}\left(c=1.0, \mathrm{CHCl}_{3}\right)$; UV (Hexane): $\lambda \max \mathrm{nm}(\log$ $\varepsilon)=240$ (4.08), 306 (2.27); MS m/z (\%): $164\left(\mathrm{M}^{+}, 77\right), 149$ (93), 135 (41), 121 (12), 107 (26), 96 (53), 79 (16), 68 (100), 67 (69), 53 (18), 41 (17); ${ }^{1} \mathrm{H}-\mathrm{NMR} \delta\left(\mathrm{CDCl}_{3}\right): 1.78(3 \mathrm{H}$, $\left.\mathrm{s},=\mathrm{CCH}_{3}\right), 2.16\left(2 \mathrm{H}, \mathrm{m}, \mathrm{CH}_{2} \mathrm{CH}_{2} \mathrm{CH}\right), 2.45(1 \mathrm{H}, \mathrm{dtd}, J=18.9$, 7.5, $\left.2.3 \mathrm{~Hz}, \mathrm{CH}_{2} \mathrm{CH}_{2} \mathrm{C}=\right), 2.65(1 \mathrm{H}, \mathrm{dtd}, J=18.9,4.8,1.0 \mathrm{~Hz}$, $\left.\mathrm{CH}_{2} \mathrm{CH}_{2} \mathrm{C}=\right), 3.13\left(1 \mathrm{H}, \mathrm{t}, J=7.6 \mathrm{~Hz}, \mathrm{CH}_{2} \mathrm{CHC}=\mathrm{O}\right), 4.77(1 \mathrm{H}$, br. $\left.\mathrm{s},=\mathrm{CH}_{2}\right), 5.00\left(1 \mathrm{H}, \mathrm{dq}, J=1.4,1.4 \mathrm{~Hz},=\mathrm{CH}_{2}\right), 6.58(1 \mathrm{H}$, $\mathrm{dd}, J=2.3,1.2 \mathrm{~Hz}, \mathrm{C}=\mathrm{CHC}=\mathrm{O}), 9.79(1 \mathrm{H}, \mathrm{s}, \mathrm{CHO}) ;{ }^{13} \mathrm{C}-$ NMR $\delta\left(\mathrm{CDCl}_{3}\right): 20.6\left(\mathrm{CH}_{3}\right), 21.0\left(\mathrm{CH}_{2}\right), 27.0\left(\mathrm{CH}_{2}\right), 55.5$ $(\mathrm{CH}), 114.3\left(\mathrm{CH}_{2}\right), 139.2(\mathrm{CH}), 142.1(\mathrm{C}), 153.8(\mathrm{C}), 194.2$ (CHO), $200.5(\mathrm{C}=\mathrm{O})$.

\subsection{4. (-)-(R)-Isorobinal $[(R)-1]$}

In the same manner as described above, $(R)-9$ (95 $\mathrm{mg}$, $0.57 \mathrm{mmol})$ gave $(R)-\mathbf{1}(67 \mathrm{mg}, 71 \%)$. Its GC/MS, ${ }^{1} \mathrm{H}-$ and ${ }^{13} \mathrm{C}$-NMR spectra were identical to those of the $S$ isomer. $[\alpha]_{\mathrm{D}}^{25}-45^{\circ}\left(\mathrm{c}=1.3, \mathrm{CHCl}_{3}\right)$. 


\section{Results and Discussion}

\section{Synthesis of both isomers}

Our synthetic route is summarized in Fig. 1. Commercially available $(R)$-perillyl alcohols $[(R)$-2] were separately epoxidized with MCPBA to give corresponding diastereomeric mixtures of $(R)-3$. The phenylselenide anion opened the mixture of $(R)-3$ to the hydroxy selenide $(R)-4$ and $(R)-5,{ }^{6)}$ which could be separated by silica gel column chromatography. The primary hydroxy group of hydroxy selenide $(R)-4$ was protected by acetylation and the resulting acetate $(R)-6$ was oxidized by excess hydrogen peroxide to effect the oxidative elimination reaction, ${ }^{6}$ giving the desired $(R)-7$. Oxidation of the allylic tertiary alcohol $(R)-7$ with 5 equivalent of PCC afforded the transposed $\alpha, \beta$-unsaturated ketone $(S)-\mathbf{8}^{7,8)} \mathrm{Fi}-$ nally, the ketone $(S)-\mathbf{8}$ was hydrolyzed with potassium carbonate at $-15^{\circ} \mathrm{C}$ to give the primary alcohol $(S)-9$. The alcohol $(S)-9$ was subsequently oxidized with Dess-Martin periodinane ${ }^{9,10)}$ to give the target molecule 1 . The overall yield of $(+)-(S)$-isorobinal $\left\{(S)-\mathbf{1},[\alpha]_{\mathrm{D}}^{28}+51^{\circ}\left(c=1.0, \mathrm{CHCl}_{3}\right)\right\}$ was $17 \%$ based on $(R)$-perillyl alcohol $(R)-2$. In the same manner, $(-)-(R)$-isorobinal $\left\{(R)-\mathbf{1},[\alpha]_{\mathrm{D}}^{25}-45^{\circ}\left(c=1.3, \mathrm{CHCl}_{3}\right)\right\}$ was synthesized from $(S)$-perillyl alcohol $(S)$-2 with similar overall yield. The GC/MS and ${ }^{1} \mathrm{H}-\mathrm{NMR}$ spectra of $(S)$ - and $(R)-\mathbf{1}$ were identical with that of the natural 1 reported by Sakata et $a l .^{2)}$

Three reaction steps from PCC oxidation of $(R)$ - and $(S)-7$ involving the concurrent allylic rearrangement of the hydroxy

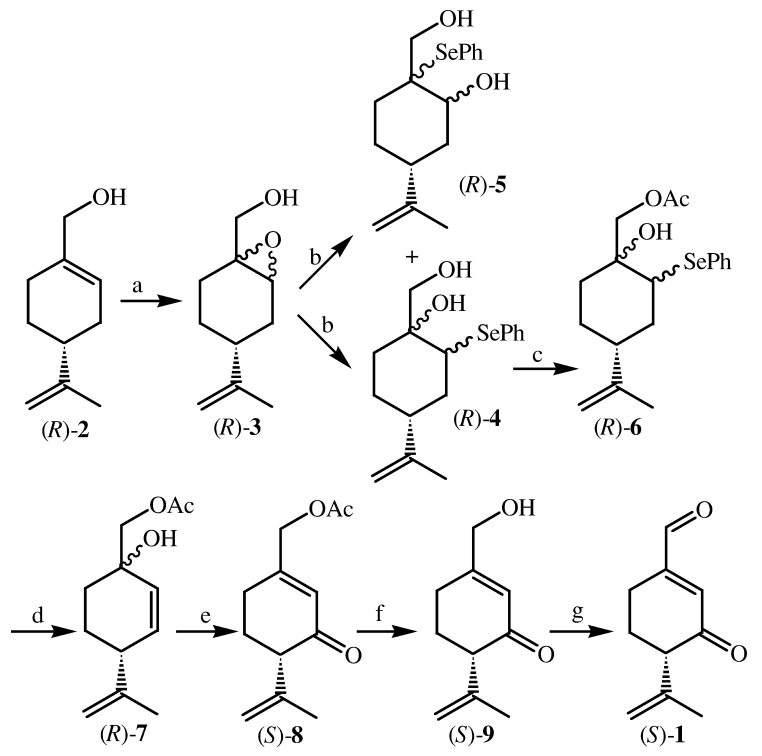

Fig. 1. Synthetic procedure of $(+)-(S)$-isorobinal $[(S)-1]]$ from $(R)-$ perillyl alcohol $[(R)-2]$.

Reagents: (a) MCPBA, $\mathrm{CH}_{2} \mathrm{Cl}_{2}$, (b) $\mathrm{Ph}_{2} \mathrm{Se}_{2}, \mathrm{NaBH}_{4}, \mathrm{EtOH}$, (c) $\mathrm{Ac}_{2} \mathrm{O}$, pyridine, (d) $30 \% \mathrm{H}_{2} \mathrm{O}_{2}, \mathrm{THF}, \mathrm{H}_{2} \mathrm{O}$, (e) $\mathrm{PCC}, \mathrm{CH}_{2} \mathrm{Cl}_{2}$, (f) $\mathrm{K}_{2} \mathrm{CO}_{3}$, $\mathrm{MeOH}$, (g) Dess-Martin periodinane, $\mathrm{CH}_{2} \mathrm{Cl}_{2}$. Following the same procedure, $(-)-(R)$-isorobinal $[(R)-1]$ was prepared from $(S)$-perillyl alcohols $[(S)-2]$.

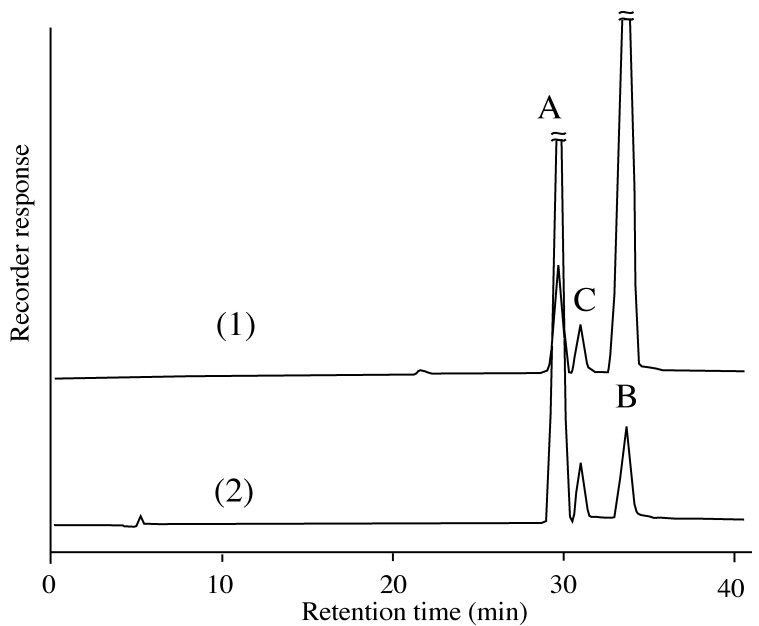

Fig. 2. Typical separation of $(R)$ - and $(S)$-isorobinal by HPLC using a chiral column. (1) Preparation of (-)-(R)-isorobinal, (2) Preparation of $(+)-(S)$-isorobinal.

A: (+)-(S)-Isorobinal, B: $(-)-(R)$-Isorobinal, C: Robinal

group to $(S)$ - and $(R)-\mathbf{1}$, via hydrolysis and Dess-Martin periodinane oxidation, proceeded successfully to give $50 \%$ yield, possibly because all reactions were carried out in nearly neutral conditions. On the other hand, the reported two step reactions involving the same PCC oxidation resulted in 38\% yield, because the next deprotection step by aq. HF in acetonitrile resulted in a poor yield $(41 \%){ }^{5)}$

\section{Enatiomeric purities and stability of isorobinal}

Enantiomeric purities of $(S)$ - and $(R)-\mathbf{1}$, determined by HPLC analyses using a chiral column (Chiralpak AD-H), were 95\% ee and $88 \%$ ee, respectively, corresponding to slightly lower $[\alpha]_{\mathrm{D}}^{25}$ values than those reported. ${ }^{5)}$ At present, it remains obscure why such a fairly large difference in ee values is observed between two enantiomers. It is possible that the optical purity of each starting perillyl alcohol $[(S)$ - and $(R)-2]$ was different, although their ee values were not determined.

The chemical structures of both $(S)$ - and $(R)-\mathbf{1}$ contained acid- and base-sensitive $\beta, \gamma$-double bond easily convertible to the more stable $\alpha, \beta$-position, and, therefore, both compounds seemed to be labile to give robinal (3-oxo-4-isopropylidene-1cyclohexene-1-carboxaldehyde). ${ }^{11)}$ As indicated in Fig. 2, a small amount of robinal is always accompanied with $(S)$ - and (R)-1 preparations.

\section{Determination of stereochemistry and its distribution among related mites}

The chiral HPLC profiles (Fig. 3) of both enantiomers will enable us to determine the absolute configuration of natural $\mathbf{1}$ of the other mite. Compound $\mathbf{1}$ is known to be distributed in the following seven species belonging to the family Acaridae among 61 species consisting of 10 families of Astigmata examined; Rhizoglypus robini, R. stosus, Rhizoglyphus sp. “oki”, Rhizoglyphus sp. "mori” [447 bps (code name RH 34, 


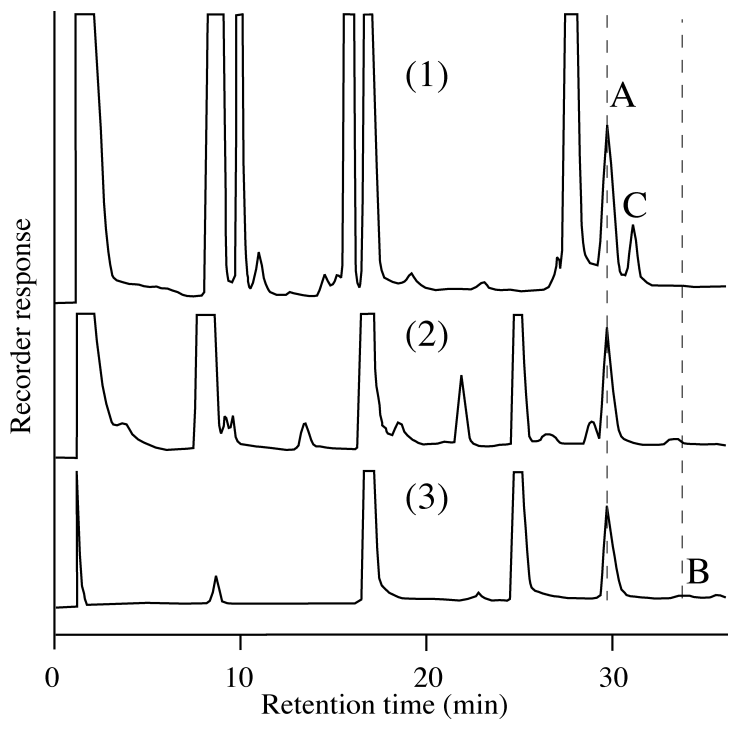

Fig. 3. Stereochemistry of natural isorobinal present in three $R h i$ zoglyphus species. (1) Rhizoglyphus sp. "mori”, (2) Rhizoglyphus sp. "oki”, (3) R. setosus.

A: (+)-(S)-Isorobinal, B: (-)-(R)-Isorobinal, C: Robinal

GenBank Accession Number: AB104969)], Tyrophagus similis, Tyroborus lini, and Schwiebea araujoae. ${ }^{1)}$ Distribution, therefore, seems to be restricted only among species belonging to the family Acaridae.

As mentioned in the Introduction, compound (1) has been identified from the unidentified Rhizoglyphus sp. "oki", without any biological activity, and was then re-identified as the sex pheromone of $R$. setosus, together with its $S$ stereochemistry. ${ }^{4)}$ In this study, the stereochemistry of $\mathbf{1}$ present in each corresponding extract of two species, the unidentified Rhizoglyohus sp. "oki" and Rhizoglyohus sp. "mori”, was determined to be $S$, though the possible presence of a minute amount of $(R)-1$ was not excluded, as indicated in Fig. 3.

In the case of $R$. robini, the content of $\mathbf{1}$ was too low to ob- tain a reliable peak by chiral HPLC analysis, and its stereochemistry remained obscure (data not indicated). Likewise, in the other three species, Tyrophagus similis, Tyroborus lini, and Schwiebea araujoae, their GC responses of $\mathbf{1}$ were too low, and no attempts were made to determine their stereochemistry.

\section{Acknowledgments}

This study was partly supported by a grant-in-aid for scientific research from the Ministry of Education, Science, Sports and Culture of Japan (Nos. 08406010, 09556010, 09876091 and 8956). N. S. is the recipient of a research fellowship (No. 01761) from the Japan Society for the Promotion of Science for Young Scientists.

\section{References}

1) Y. Kuwahara: "Advances in Insect Chemical Ecology," ed. by R. T. Cardé and J. G. Millar, Cambridge University Press, Cambridge, pp. 76-109, 2004.

2) T. Sakata, Y. Kuwahara and K. Kurosa: Naturwissenschaften 83, 427 (1996).

3) K. Noge, N. Mori, C. Tanaka, R. Nishida, M. Tsuda and Y. Kuwahara: Exp. Appl. Acarol. 35, 29-46 (2005).

4) A. Mizoguchi, K. Murakami, N. Shimizu, N. Mori, R. Nishida and Y. Kuwahara: Exp. Appl. Acarol. 36, 107-117 (2005).

5) T. Liang and S. Kuwahara: Biosci. Biotech. Biochem. 66, 2501-2503 (2002).

6) K. B. Sharpless and R. F. Lauer: J. Am. Chem. Soc. 95, 2697-2699 (1973).

7) G. W. Dauben and M. D. Michno: J. Org. Chem. 42, 682-685 (1977).

8) K. Domon and K. Mori: Eur. J. Org. Chem. 2000, 3783-3785 (2000).

9) D. B. Dess and J. C. Martin: J. Am. Chem. Soc. 113, 7277-7287 (1991).

10) M. Takagi, H. Takikawa and K. Mori: Biosci. Biotechnol. Biochem. 65, 2065-2069 (2001).

11) W. S. Leal, Y. Kuwahara and T. Suzuki: Naturwissenschaften 77, 387-388 (1990). 